\title{
Common Sources of Inflammation and Their Impact on Hematopoietic Stem Cell Biology
}

\author{
Daniel Hormaechea-Agulla ${ }^{1}$ Duy T. Le ${ }^{1,2} \cdot$ Katherine Y. King ${ }^{1,2}$
}

(C) Springer Nature Switzerland AG 2020

\begin{abstract}
Purpose of Review Inflammatory signals have emerged as critical regulators of hematopoietic stem cell (HSC) function. Specifically, HSCs are highly responsive to acute changes in systemic inflammation and this influences not only their division rate but also their lineage fate. Identifying how inflammation regulates HSCs and shapes the blood system is crucial to understanding the mechanisms underpinning these processes, as well as potential links between them.

Recent Findings A widening array of physiologic and pathologic processes involving heightened inflammation are now recognized to critically affect HSC biology and blood lineage production. Conditions documented to affect HSC function include not only acute and chronic infections but also autoinflammatory conditions, irradiation injury, and physiologic states such as aging and obesity.

Summary Recognizing the contexts during which inflammation affects primitive hematopoiesis is essential to improving our understanding of HSC biology and informing new therapeutic interventions against maladaptive hematopoiesis that occurs during inflammatory diseases, infections, and cancer-related disorders.
\end{abstract}

Keywords Hematopoiesis $\cdot$ Pro-inflammatory cytokines $\cdot$ Infectious diseases $\cdot$ Bone marrow $\cdot$ Inflammatory conditions

\section{Introduction}

Hematopoietic stem cells (HSCs) are the most primitive hematopoietic population resident in the bone marrow (BM). Although a very rare population, HSCs are the foundation of the hematopoietic tree. Through self-renewal and differentiation into other progenitor populations, HSCs have the unique ability to completely regenerate the blood system after transplantation and thus have been the focus of heavy study for many decades. HSCs are also the foundation of many hematological disorders and pathologies, since any mutation that occurs in an HSC will be passed to downstream blood

Daniel Hormaechea-Agulla and Duy T. Le contributed equally to this work.

This article is part of the Topical Collection on Cell Maintenance

Katherine Y. King

kyk@bcm.edu

1 Section of Infectious Diseases, Department of Pediatrics, Baylor College of Medicine, Houston, TX, USA

2 Program in Immunology, Graduate School of Biomedical Sciences, Baylor College of Medicine, Houston, TX, USA lineages. Therefore, it is of critical importance to understand the myriad processes that directly or indirectly affect HSC biology.

HSCs were long perceived to be a population protected from external signals, but recent overwhelming evidence has shifted that view $[1 \bullet \bullet$. For instance, inflammation is now well recognized to have an important influence on HSC function $[2 \bullet \bullet, 3 \bullet]$. Many types of endogenous and exogenous factors, ranging from mechanical/thermal stimuli to pathogens or aging, induce local or systemic inflammation, and each of these has the potential to affect HSC activity. The aim of this review is to summarize the pro-inflammatory mediators and sources of inflammation known to affect HSC biology.

\section{Pro-inflammatory Cytokines}

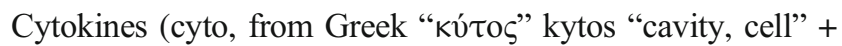

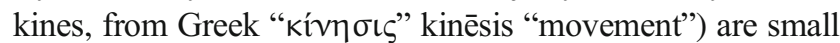
proteins $(5-20 \mathrm{kDa})$ that signal between cells and in many cases can mediate inflammatory responses. Cells with innate receptors sense a range of inflammatory insults (i.e., tissue damage, infection) and in response produce pro- 
inflammatory cytokines to recruit immune cells to clear the damage.

HSCs express toll-like receptors (TLRs) that recognize pathogen-associated molecular patterns (PAMPs) and can therefore directly sense pathogens $[1 \bullet \bullet, 4 \bullet]$. Critically, on their membrane, HSCs also express cytokine/growth factor receptors that are able to sense pro-inflammatory mediators secreted by other cells $[1 \bullet \bullet$. Though the role of pro-inflammatory cytokines on HSC regulation is well known and widely accepted

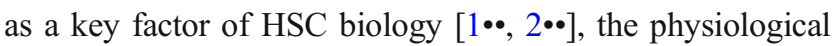
relevance of direct sensing and responses to pathogens (by being infected or the recognition of PAMPs) by HSCs remains unclear $[4 \bullet, 5 \bullet]$. Specifically, the consequences of inflammation on HSC persistence, clonal evolution, differentiation, and function remain the subject of current investigations.

Distinguishing between the acute and chronic effects of pro-inflammatory cytokines on HSC function represents another layer of complexity. While short-term induction by a range of cytokines such as IFN $\gamma, \operatorname{IFN} \alpha / \beta, \mathrm{IL}-1, \mathrm{TNF} \alpha$, or IL-6 (Table 1) increases proliferation, long-term exposure to these same cytokines can damage HSC function [2••]. Recently, the impact of pro-inflammatory cytokines on HSCs has been thoroughly reviewed [2••], as summarized in Table 1 . The impact of inflammatory cytokines on the HSC niche has also been reviewed recently $[3 \cdot 6]$.

The sources of pro-inflammatory cytokines that affect HSC function range widely, from acute and chronic infections to radiation injury and autoinflammation (Table 1). These sources of inflammation and conditions in which they affect HSC biology are the main focus of this review.

\section{Sources of Inflammation}

\section{Infectious Diseases}

\section{Bacterial Infections}

Mycobacterial infections are associated with bone marrow suppression, perhaps because of their chronicity and capacity to invade the bone marrow. Our group had shown that mycobacterial infections such as those mediated by Mycobacterium avium promote HSC proliferation in an IFN $\gamma$-dependent manner $[7 \bullet \bullet]$, ultimately depleting HSCs due to terminal myeloid differentiation promoted by the transcription factor Batf2 [8•, 9]. Similarly, chronic Mycobacterium tuberculosis (Mtb) infection drives IFN $\gamma$ production in CD4 T cells, thus disrupting hematopoietic homeostasis [10]. Moreover, Choi et al. demonstrated during Mtb infection in mice that HSPC proliferation is associated with increased TNF $\alpha$ and IL- 6 signaling via the toll-like receptor (TLR) pathway [11].

Indeed, HSCs express TLR2 and TLR4 and therefore are able to recognize bacterial cell wall components from Gram- positive and Gram-negative bacteria, respectively [1••]. Mice exposed to TLR2 and TLR4 antagonists showed increased HSC differentiation and reduced self-renewal capacity [4•, 12•]. LPS, a TLR4 agonist, affects HSC function through TLR4-TRIF-ROS-p38 signaling [4•]. A single dose of LPS increases the BM serum levels of IL-1ß, IL- $1 \alpha$, and CXCL9 [13]. Chronic low-dose LPS administration increases HSC proliferation and myeloid differentiation and functionally impairs HSCs, as shown by their reduced repopulation capacity in serial transplantation experiments [14•, 15]. Sonic Hedgehog (SHH) signaling has also been shown to play a role in HSPC activation during Escherichia coli infection [16].

Using a mouse model of an emerging tick-borne disease carried by the lone star tick Amblyomma americanum, infection by the Gram-negative bacterium Ehrlichia muris was shown to suppress BM function and myelopoiesis in an IFN $\gamma$-dependent manner [17-20]. Moreover, Smith et al. demonstrated that during $E$. muris infection, IFN $\alpha / \beta$ signaling also induces BM suppression, leading to emergency myelopoiesis and reduced hematopoiesis. Consistent with this, Ifnar1 null mice had increased hematopoiesis and maintained HSC functions despite infection with E. muris. Mechanistically, it has been reported that type I IFNs induce loss of HSPC via RIPK1-dependent cell death during E. muris infection [21].

In Gram-positive Staphylococcus aureus infection, HSPCs are stimulated by IL-1ß traffic to the infection site, where they are activated via TLR2/MyD88 and produce prostaglandin E2 (PGE2) to promote granulopoiesis [22•, 23]. Another study showed that treatment with TLR2 agonist $\mathrm{PAM}_{3} \mathrm{CSK}_{4}$ caused HSC expansion but loss of their BM repopulating activity; these effects were partially mediated by G-CSF and TNF $\alpha$ [12•].

\section{Viral Infections}

Viral infections, including parvovirus [24, 25], HIV [26], viral hepatitis [27], Epstein-Barr virus [28]), and influenza [29], are often associated with BM suppression due to increased production of pro-inflammatory cytokines [30]. Our group has shown that interferons released during LCMV infection promote cell division and myeloid differentiation of HSPCs [9]. Though both type I and type II interferons are induced during LCMV infection, effects on HSPCs were especially dependent on type II interferon. Similarly, vesicular stomatitis virus (VSV) and murine cytomegalovirus (MCMV) infections activate LT-HSC independently of type I interferon receptor signaling [31•]. Therefore, strong evidence in LCMV, VSV, and MCMV virus models showed that type II interferon but not type I interferon is necessary for HSPC activation. Non-acute MCMV infection induces a sustained inflammatory milieu within the BM that is associated with long-lasting impairment of LT-HSC function, even when the virus is cleared from BM 


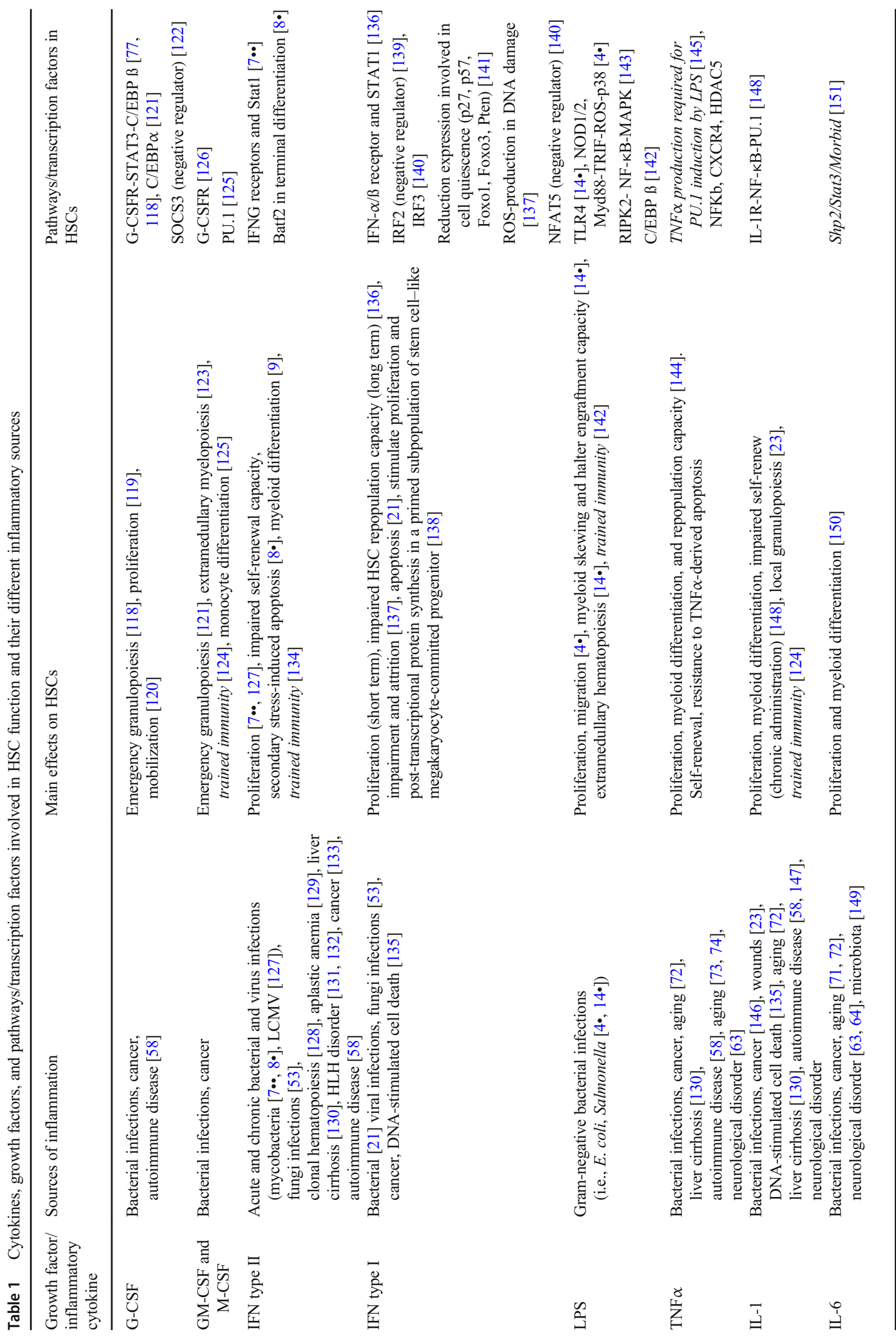


[31•]. Moreover, HIV infection can alter HSPC differentiation via ILR8 signaling [32]. IL-18, a cytokine that induces IFN $\gamma$ production, protects lymphoid HSPC from apoptosis during primary HIV infection; however, high levels of IL-18 in chronic HIV infection deter lymphoid HSPC proliferation $[33,34]$. Of interest, SARS-CoV-2 infection (the virus that causes COVID-19) also produces a dramatic increase in many pro-inflammatory cytokines known to affect HSCs, such as TNF, IL-1ß, IL-6, IL-8, G-CSF, and GM-CSF. This increase occurs especially in patients who are critically ill with COVID-19; therefore, future studies should investigate how this novel coronavirus affects $\mathrm{BM} / \mathrm{HSC}$ activity [35].

Some miRNAs have been associated with HSC regulation in viral infections $[36,37]$. Our group reported that the loss of miR-22, which normally promotes IFN $\alpha$ production during viral infection, is beneficial for mice in a model of LCMV infection. We found that miR-22-null mice had better overall survival and HSC homeostasis than WT mice after LCMV challenge, which can be attributed to their blunted IFN response to infection [37].

\section{Protozoan and Fungal Infections}

Plasmodium is a genus of intracellular parasites that cause malaria. Using a mouse model of malaria infection based on natural, sporozoite-driven Plasmodium berghei infection, Vainieri et al. showed that HSCs become highly proliferative during infection [38•]. In line with this increase in proliferation, LT-HSC and LMPP numbers were higher as early as day 3 post-infection indicating that critical responses take place in the primitive hematological populations at very early stages of sporozoite infection [38•]. Furthermore, primitive HSCs proliferated significantly at days 7-10 of infection. Given that replication stress has been associated with loss of functionality of aged HSCs [39] and the appearance of pre-malignant hematopoietic conditions such as clonal hematopoiesis [40], results from this study support the long-term consequences of sustained HSC proliferation on HSC fitness.

Toxoplasma gondii (T. gondii), the agent of toxoplasmosis, is a widespread intracellular protozoan parasite that infects up to a third of the world's population [41]. Given its overall prevalence, reactivation of toxoplasmosis is a significant side effect among recipients of hematopoietic stem cell transplantation (HSCT) [42]. T. gondii infection leads to bone marrow suppression, reducing white blood cells, reticulocytes, and platelets [43]. Mice with $T$. gondii infection showed increased granulopoiesis, leading to increased functional activity of granulocytes in the blood [43]. T. gondii GA15 protein has been shown to be involved in the regulation of TNF and NF$\mathrm{kB}$ signaling pathways using hamster kidney cells in vitro [44] and triggers cGAS/STING signaling in a STING- and TRAFdependent manner in mice [45]. Since activation of STING is necessary for the production of type I interferons [46] and
TRAF pathways affect primitive hematopoiesis in other models [47], these signaling pathways also may affect hematopoiesis during toxoplasmosis infection in humans.

Fungal infection is often associated with high mortality in HSCT patients [48, 49]. After stem cell transplant, fungal infection, including mucormycosis, can lead to myriad severe complications including invasive fungal sinusitis, emphysematous gastritis, and other invasive fungal infections associated with extremely high mortality $[50,51]$. Mucormycosis is commonly found in hematological patients, according to a review of 929 reported cases by Roden et al. [52]. Although it has been reported that IFN $\gamma$ signaling directly activates antifungal responses in neutrophils [53], we found no evidence that Aspergillus fumigatus infections affect HSC function (data not published); further research is warranted to deepen our knowledge in this area.

\section{Chronic Inflammatory/Autoimmune Diseases/Neurological Diseases}

Abnormal and steady elevation of serum pro-inflammatory cytokines such as IL-6, TNF $\alpha$, IL-17 $\alpha$, GM-CSF, and GCSF that potentially affect HSC function is a hallmark of chronic inflammatory and autoimmune diseases. Chronic intestinal inflammation, driven by abnormal IL-23-dependent responses, causes increased proliferation of HSPCs, which accumulate at extramedullary sites. Eosinophil activation by GM-CSF, which promotes eosinopoiesis in synergy with IL5 , is responsible for this dysregulated hematopoiesis [54・,55]. Niu et al. have shown in a lupus mouse model that HSCs expanded with an increased capacity for self-renewal due to a single nucleotide polymorphism in the $c d k n 2 c$ gene, leading to downregulation of $\mathrm{p} 18^{\mathrm{INK} 4 \mathrm{c}}\left[56^{\circ}\right]$. Moreover, in patients with atherosclerotic cardiovascular disease (CVD), HSPCs have higher proliferative capacity than healthy individuals [57]. Chronic inflammation also is associated with autoimmune diseases such as rheumatoid arthritis (RA). In one recent report using a mouse model of human RA, Hernandez et al. showed that HSCs in RA mice persist in a quiescent state, consistent with the activation of proliferation arrest genes despite the increase of inflammation and myeloid production. Treating RA mice with inflammatory cytokine blockade normalized hematopoiesis and attenuated myelopoiesis [58]. Some monogenic autoimmune disorders such as IPEX syndrome and Stat 1 gain-of-function are characterized by excessive inflammation and disordered hematopoiesis [59, 60]. With increasing evidence suggesting chronic inflammation disrupts HSC functions, HSCT has become a viable option to treat patients with such severe chronic inflammatory and autoimmune diseases [61].

In addition to traditional autoimmune diseases, people with neurological disorders including depression have been shown to present with higher serum levels of TNF $\alpha$, IL-6, IL-13, IL- 
18, IL-12, IL-1RA, and sTNFR2, as well as decreased IFN $\gamma$ [62-64]. Mechanistically, activation of microglia through PAMPs or danger-associated molecular patterns (DAMPs) signaling promotes inflammatory responses in neurological disorders such as Alzheimer's disease, frontotemporal dementia, and Parkinson's disease [65]. Since many of the cytokines associated with neuroinflammation are also involved in HSC regulation (Table 1), it is reasonable to speculate that people with neuroinflammatory disorders could have BM/HSC abnormalities. Interestingly, neurotrophic factor receptor RET plays a role in promoting HSC survival and expansion by activation of p38/MAP and CREB through Bcl2/Bcl211 [66]. Moreover, HSC quiescence is regulated by Schwann cells - a type of glia wrapping nerve fibers in the BMthrough TGF- $\beta$ signaling, suggesting that glial cells may maintain HSC hibernation by regulating activation of latent TGF- $\beta$ within the BM niche [67]. Due to the high and increasing prevalence of these disorders, new studies are needed to reveal the plausible relationship between inflammation, neurological diseases, and HSC function.

\section{Aging}

Many alterations that occur in the aging hematopoietic compartment are common to inflammatory processes $[3 \cdot, 68]$, including (1) myeloid bias in hematopoiesis with a shift in the frequency of CD150+ high HSCs [69], (2) accumulation of DNA damage [70], and (3) increased basal levels of proinflammatory cytokines such as IL-6 [71, 72], TNF- $\alpha$ [73, 74], IL-1R $\alpha$, and C-reactive protein [3•] in the serum of healthy elderly people. Chronic treatment with a low dose of LPS in mice leads to myeloid skewing [14•] reminiscent of the myeloid skewing that is seen in normal aging. A recent report linked the activity of retrotransponsable elements (RTE) with sterile inflammation to aging. During cellular senescence, LINE-1 - the only human RTE capable of autonomous retro transposition - became transcriptionally derepressed and activated an IFN-I response, which further contributed to the maintenance of the senescence-associated secretory phenotype [75]. Environmental factors including inflammation are also important in the progression of age-associated clonal hematopoiesis [76•], as shown by high levels of IFN $\gamma$ in the serum of patients with DNMT3A-related clonal hematopoiesis and ulcerative colitis [77]. Collectively, these studies reveal a clear interplay between low-grade inflammation and aging and suggest that the hematopoietic changes seen with age are attributable at least in part to inflammation.

\section{Chemotherapy and Radiation}

Under the stress caused by exposure to cytotoxic chemotherapy, HSCs are activated and enter the cell cycle, which drives emergency myelopoiesis and rapid regeneration of the hematopoietic system. Moreover, radiation including X-rays and $\gamma$-rays induce DNA damage, reactive oxygen/nitrogen species, ER stress, and hypoxia, triggering inflammatory responses and increased production of pro-inflammatory cytokines, such as IL- $1 \alpha$, IL- $1 \beta$, IL-12p 40 , TNF- $\alpha$, and IFN $\gamma$ [78]. Radiation can also lead to genetic changes in HSCs [79]. Rodman et al. reported that exposure to chronic solar energetic particles (SEP) and galactic cosmic ray (GCR) radiation can cause mutations in genes involved in hematopoiesis, modulate the engraftment as well as lineage commitment of HSCs, damage BM stromal cells and thus disrupt the niche, and contribute to the development of abnormal T-ALL [80, 81].

\section{Gender}

Physiological differences between women and men play a prominent role in their exposure to infectious diseases and the frequency and manifestations of autoimmunity, including autoimmune diseases [82]. It is widely accepted that men are more susceptible to infections (i.e., tuberculosis and parasites), while women present with more autoimmune diseases [82]. Estrogen and testosterone differentially regulate inflammation [83]. Powell et al. revealed that estrogen increased inflammation in a model of human knee joint injury, while testosterone reduced inflammation at the site of injury [83]. Sex hormones regulate the transcription of many genes involved in immune cell development and maturation, regulation of immune responses, and modulation of immune signaling pathways; however, few reports have studied the direct effect of sex hormones on HSC regulation [82]. Nakada et al. showed that estrogen can induce more frequent HSC division in females compared with males through its interaction with estrogen receptor-alpha on HSCs [84••]. Indeed, this study showed estradiol treatment increased HSC division in male mice.

\section{Lifestyle Factors Can Affect HSC Inflammation}

\section{Dietary}

Metabolic disorders including atherosclerosis, obesity, and type 2 diabetes are characterized by the presence of a chronic inflammatory state that negatively affects the regulation and function of HSCs and progenitors [85]. Therefore, human behaviors/actions to reduce those deleterious, chronic sources of inflammation are vital to improve HSC function. It has been reported that the ability for HSCs to self-renew and proliferate depends on metabolism [86-88]. While fasting appears to enhance HSC function, a number of recent reports have found that high-fat diets (HFDs) and obesity reduce HSC activity. HFDs are associated with increasing inflammation in the central nervous system, liver, adipose tissue, skeletal muscle, and 
intestine [89]; alter the microbiome; increase the level of LPS; and promote production of pro-inflammatory cytokines such as IL- $1 \beta$, IL-6, and TNF- $\alpha$ [90]. Accumulation of cholesterol in innate immune cells [91] and HSPC [92] promoted increased inflammatory responses through the TLR signaling pathway [91] and increased myelopoiesis by amplifying Janus kinase 2 (JAK2) signaling [92]. Indeed, inhibition of JAK2 reduced atherogenesis by suppressing myelopoiesis [93].

Vitamins are essential nutrients for human health. Taya et al. showed that valine amino acid plays an important role in the maintenance of HSC and BM niche [94]. In a recent report, vitamin $\mathrm{B}_{6}$ intake reduced the number of inflammatory markers, such as C-reactive protein, IL-6 receptor, and white blood cell count as well as overall inflammation score [95]. Vitamin $\mathrm{C}$ alleviates inflammation by decreasing the level of IL-6, fasting blood glucose, and high-sensitivity C-reactive protein [96], and regulates HSPC self-renewal by acting as a co-factor for TET2 by driving DNA hypomethylation. Vitamin D treatment has also been shown to promote colony formation by HSPCs [97].

A healthy diet is associated with increased survival and lower risks of chronic health conditions in HSCT patients [98]. In line with this, a diet rich in n-3 PUFA-rich fish oil promotes hematopoiesis in the bone marrow and spleen of mice, mediated in part by MMP12, and may induce myeloid-derived suppressor cell differentiation to suppress tissue inflammation [99]. Additionally, a number of studies support the idea that fasting/dietary restriction plays an important role in prolonging a healthy lifespan by regulating inflammatory responses and HSC function $[100 \cdot, 101]$. Cheng et al. showed that 48 -h cycles of fasting promoted $\mathrm{HSC}$ regeneration in mice by inhibiting insulin growth factor 1 (IGF1) signaling [100•]. Diabetes is also seen as fasting-like state. A retrospective analysis of HSCT patients found that diabetes negatively correlated with donor HSPC mobilization [101].

\section{Exercise}

Frodermann et al. reported that exercise modifies HSPC function by reducing leptin signaling in the bone marrow niche $[102 \bullet \bullet$. Specifically, physical activity can protect mice from chronic leukocytosis by reducing hematopoietic activity without compromising emergency hematopoiesis. Exercise attenuates the production of leptin in adipose tissue, which in turn enhances CXCL12, a quiescence-promoting hematopoietic niche factor important in the maintenance of HSC function [102••]. In a separate study, mice fed a high-fat diet without exercise showed increased common myeloid progenitor cells and BM inflammation [103]. Overall, these studies indicate that exercise maintains HSC function by decreasing inflammation within the $\mathrm{BM}$ niche.

\section{Smoking}

Mice exposed to cigarette smoke exhibited increased extramedullary hematopoiesis in the spleen, inhibition of HSPC homing into BM, decreased mesenchymal stromal cells and HSCs, and increased pro-proliferation genes that lead to the expansion of HSPCs [104-106]. Cigarette smoke extract decreased bone formation but increased an array of different of interleukins such as IL-1 $\beta$, IL-2, IL4, IL-5, IL-9, IL-10, IL-12p40, IL-13, IL-17- $\alpha$, G-CSF, GM-CSF TNF- $\alpha$, and IFN- $\gamma[107 \bullet \bullet]$. Since many of these cytokines have been shown to impact hematopoiesis, it is reasonable to hypothesize that smoking may significantly affect hematopoiesis by inducing systemic inflammation. In support of these effects, smoking has been identified as one of the factors related to the emergence of clonal hematopoiesis [76•] and hematological cancers [108•].

\section{Microbiome/Antibiotics}

Human conditions associated with altered intestinal bacterial populations, such as inflammatory bowel syndrome or prolonged antibiotic use, are associated with adverse hematologic effects, including anemia and neutropenia [109]. Recent studies into these interactions illustrated that microbiota modulate hematopoiesis in the BM [109]. Germ-free mice have fewer HSPCs, abnormal splenic myeloid counts, and impaired T cell function compared with wild-type mice $[110,111,112 \cdot \bullet, 113-115]$. Our group has shown that mice treated with antibiotics have reduced red blood cells, platelets, and white blood cells in peripheral blood $[109,112 \cdot \bullet]$, coupled with decreased HSCs, multipotent progenitors, granulocytes, and $\mathrm{B}$ cells in the BM. However, these studies also revealed an increase in the number of $\mathrm{CD} 8+\mathrm{T}$ cells, suggesting disruption of $\mathrm{T}$ cell homeostasis in antibiotic-treated mice could lead to increased inflammation and cause impaired progenitor maintenance [112••]. Mechanistically, Stat1null mice and antibiotic-treated wild-type mice had similarly low numbers of BM HSPCs and granulocytes. We found that treating Stat1-null mice with antibiotics did not further suppress cell counts, suggesting that STAT1 signaling, stimulated by the microbiota, is required for normal hematopoiesis and providing a clear link between microbiome and inflammatory/interferon pathways $[112 \bullet \bullet]$.

Indeed, a recent report by Staffas et al. showed the microbiome and antibiotic treatment have an even more profound effect on HSC regulation than previously envisioned. The authors found that gut microbiota induces engraftment of HSPCs following BM transplant, while antibiotic treatment impairs immune reconstitution in the post-transplant setting [116]. Moreover, Lee et al. showed 


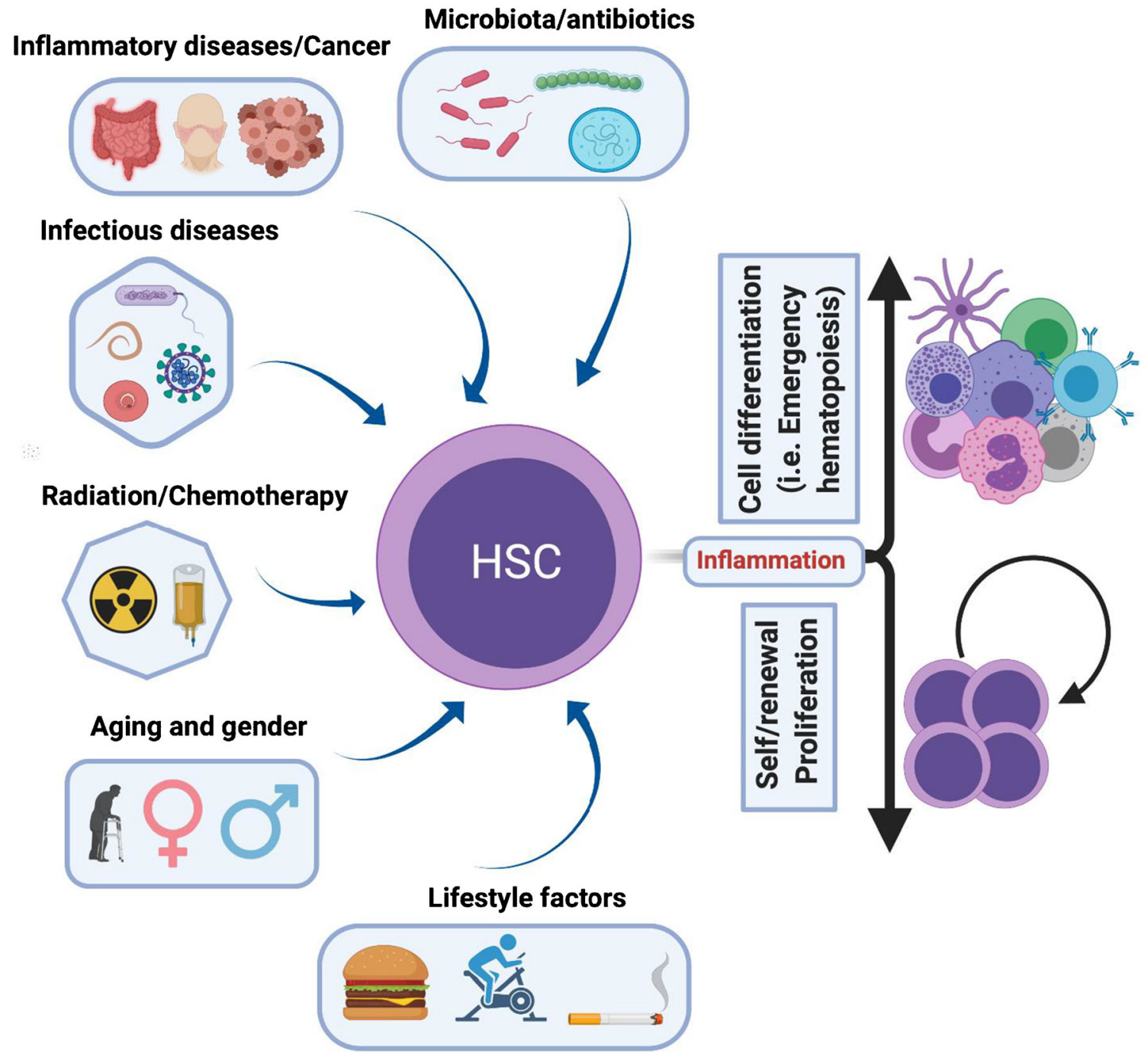

Fig. 1 Main sources of inflammation that affect HSC biology

that microbiota-derived molecules are transported in the $\mathrm{BM}$ and recognized by $\mathrm{BM} \mathrm{CX} 3 \mathrm{CR} 1+$ mononuclear cells to modulate hematopoiesis [117].

\section{Conclusions and Future Directions}

In summary, recent work indicates that many different conditions (Fig. 1) produce pro-inflammatory mediators that affect HSC biology. Rather than being inert cells that are isolated and protected from external signals, we now know that HSCs are constantly responding to the shifting inflammatory environment caused by infectious diseases, cancer, gender, aging, radiation, and lifestyle factors (Fig. 1). Inflammation is the immune system's response to harmful stimuli and initiates the healing processes and defense mechanisms vital to health. However, chronic inflammation contributes to a number of diseases and critically damages HSC biology. Since HSCs are the foundation of all blood cells and any genetic or epigenetic modification is passed from them to downstream populations, it is vital to understand how inflammation disrupts HSCs and blood regulation.

While some inflammatory sources are intrinsic to life (i.e., aging), others can be modulated (i.e., diet, smoking) to improve health. Current evidence demonstrates that a healthy lifestyle can benefit HSC biology and thereby prevent or delay the appearance of pre-malignant conditions and/or hematological disorders fueled by inflammation. Further research is necessary to deepen our understanding of how specific inflammatory insults physiologically alter HSCs and reveal the most effective therapeutic strategies to diminish the deleterious effects of inflammation on BM function.

Acknowledgments The authors would like to thank Catherine Gillespie and members of the King Lab for editing and constructive contributions to this work. 
Funding Information KYK, DHA, and DL were supported by the NIH grants R01HL136333, R01HL134880, and R01AI141716 (KYK) and T32HL092332 (DL).

\section{Compliance with Ethical Standards}

Conflict of Interest Katherine King, Daniel Hormaechea Agulla, and Duy Le declare that they have no conflict of interest.

Human and Animal Rights and Informed Consent This article does not contain any studies with human or animal subjects performed by any of the authors.

\section{References}

Papers of particular interest, published recently, have been highlighted as:

- Of importance

•. Of major importance

1.• King KY, Goodell MA. Inflammatory modulation of HSCs: viewing the HSC as a foundation for the immune response. Nat Rev Immunol. 2011;11(10):685-92. This review summarizes the effects of infection on hematopoietic stem cell function and how these effects may have a pivotal role in directing the immune response from the bone marrow.

2.• Chavakis T, Mitroulis I, Hajishengallis G. Hematopoietic progenitor cells as integrative hubs for adaptation to and fine-tuning of inflammation. Nat Immunol. 2019;20(7):802-11. This review discusses the mechanisms that govern the adaptation of hematopoietic progenitor cells to inflammation and its sequelae in the pathogenesis of human disease.

3. Kovtonyuk LV, et al. Inflamm-aging of hematopoiesis, hematopoietic stem cells, and the bone marrow microenvironment. Front Immunol. 2016;7:502. This comprehensive review present evidence supporting the concept of "Inflamm-Aging," or inflammation-associated aging of hematopoiesis and their effects on bone marrow microenvironment.

4. Takizawa H, et al. Pathogen-induced TLR4-TRIF innate immune signaling in hematopoietic stem cells promotes proliferation but reduces competitive fitness. Cell Stem Cell. 2017;21(2):225-40 e5. This study shows that while direct TLR4 activation in HSCs might be beneficial for controlling systemic infection, prolonged TLR4 signaling has detrimental effects and may contribute to inflammation-associated HSPC dysfunction.

5. Boettcher S, et al. Endothelial cells translate pathogen signals into G-CSF-driven emergency granulopoiesis. Blood. 2014;124(9): 1393-403. This elegant study shows that endothelial cells (ECs), but not hematopoietic cells, hepatocytes, pericytes, or BM stromal cells, are essential cells for emergency granulopoiesis driven by G-CSF.

6. Takizawa H, Manz MG. Impact of inflammation on early hematopoiesis and the microenvironment. Int J Hematol. 2017;106(1):27-33.

7.• Baldridge MT, et al. Quiescent haematopoietic stem cells are activated by IFN-gamma in response to chronic infection. Nature. 2010;465(7299):793-7. This is the first study to show that an increased proportion of long-term repopulating HSCs proliferate during $M$. avium infection, and that this response requires interferon-gamma (IFN-gamma) but not interferonalpha (IFN-alpha) signaling.
8. Matatall KA, et al. Chronic infection depletes hematopoietic stem cells through stress-induced terminal differentiation. Cell Rep. 2016;17(10):2584-95. This study elucidates mechanisms underlying bone marrow failure during chronic infections, which is due to impaired self-renewal and increased terminal differentiation.

9. Matatall KA, Shen CC, Challen GA, King KY. Type II interferon promotes differentiation of myeloid-biased hematopoietic stem cells. Stem Cells. 2014;32(11):3023-30.

10. Li F, Liu X, Niu H, Lv W, Han X, Zhang Y, et al. Persistent stimulation with Mycobacterium tuberculosis antigen impairs the proliferation and transcriptional program of hematopoietic cells in bone marrow. Mol Immunol. 2019;112:115-22.

11. Choi HH, Kim KK, Kim KD, Kim HJ, Jo EK, Song CH. Effects of mycobacterial infection on proliferation of hematopoietic precursor cells. Microbes Infect. 2011;13(14-15):1252-60.

12. Herman AC, et al. Systemic TLR2 agonist exposure regulates hematopoietic stem cells via cell-autonomous and cell-nonautonomous mechanisms. Blood Cancer J. 2016;6:e437. In this study, the authors indicate that systemic exposure of mice to a TLR2 agonist leads to an expansion of bone marrow and spleen phenotypic HSCs and progenitors, but a loss of HSC self-renewal capacity.

13. Hemmati S, et al. PI3 kinase alpha and delta promote hematopoietic stem cell activation. JCI Insight. 2019;5.

14. Esplin BL, et al. Chronic exposure to a TLR ligand injures hematopoietic stem cells. J Immunol. 2011;186(9):5367-75. This study shows that repeated exposure to small amounts of LPS is harmful to long-term repopulating stem cells.

15. Liu A, Wang Y, Ding Y, Baez I, Payne KJ, Borghesi L. Cutting edge: hematopoietic stem cell expansion and common lymphoid progenitor depletion require hematopoietic-derived, cellautonomous TLR4 in a model of chronic endotoxin. J Immunol. 2015;195(6):2524-8.

16. Shi X, Wei S, Simms KJ, Cumpston DN, Ewing TJ, Zhang P. Sonic Hedgehog signaling regulates hematopoietic stem/progenitor cell activation during the granulopoietic response to systemic bacterial infection. Front Immunol. 2018;9:349.

17. MacNamara KC, Racine R, Chatterjee M, Borjesson D, Winslow GM. Diminished hematopoietic activity associated with alterations in innate and adaptive immunity in a mouse model of human monocytic ehrlichiosis. Infect Immun. 2009;77(9):4061-9.

18. MacNamara KC, Oduro K, Martin O, Jones DD, McLaughlin M, Choi $\mathrm{K}$, et al. Infection-induced myelopoiesis during intracellular bacterial infection is critically dependent upon IFN-gamma signaling. J Immunol. 2011;186(2):1032-43.

19. Dumler JS. Anaplasma and Ehrlichia infection. Ann N Y Acad Sci. 2005;1063:361-73.

20. MacNamara KC, Jones M, Martin O, Winslow GM. Transient activation of hematopoietic stem and progenitor cells by IFNgamma during acute bacterial infection. PLoS One. 2011;6(12):e28669.

21. Smith JNP, Zhang Y, Li JJ, McCabe A, Jo HJ, Maloney J, et al. Type I IFNs drive hematopoietic stem and progenitor cell collapse via impaired proliferation and increased RIPK1-dependent cell death during shock-like ehrlichial infection. PLoS Pathog. 2018;14(8):e1007234.

22. Granick JL, et al. Staphylococcus aureus recognition by hematopoietic stem and progenitor cells via TLR2/MyD88/PGE2 stimulates granulopoiesis in wounds. Blood. 2013;122(10):1770-8. In this important study, the authors conclude that granulopoiesis in S. aureus-infected wounds is induced by TLR2/MyD88 activation of HSPCs through a mechanism that involves autocrine production and activity of PGE2.

23. Falahee PC, Anderson LS, Reynolds MB, Pirir M, McLaughlin $\mathrm{BE}$, Dillen CA, et al. Alpha-toxin regulates local granulocyte 
expansion from hematopoietic stem and progenitor cells in Staphylococcus aureus-infected wounds. J Immunol. 2017;199(5):1772-82.

24. Mishra B, Malhotra P, Ratho RK, Singh MP, Varma S, Varma N. Human parvovirus B19 in patients with aplastic anemia. Am J Hematol. 2005;79(2):166-7.

25. Kurtzman GJ, Ozawa K, Cohen B, Hanson G, Oseas R, Young NS. Chronic bone marrow failure due to persistent B19 parvovirus infection. N Engl J Med. 1987;317(5):287-94.

26. Zauli G, Capitani S. HIV-1-related mechanisms of suppression of CD34+ hematopoietic progenitors. Pathobiology. 1996;64(1): 53-8.

27. Brown KE, Tisdale J, Barrett AJ, Dunbar CE, Young NS. Hepatitis-associated aplastic anemia. N Engl J Med. 1997;336(15):1059-64.

28. Ahronheim GA, Auger F, Joncas JH, Ghibu F, Rivard GE, RaabTraub N. Primary infection by Epstein-Barr virus presenting as aplastic anemia. N Engl J Med. 1983;309(5):313-4.

29. Sedger LM, Hou S, Osvath SR, Glaccum MB, Peschon JJ, van Rooijen N, et al. Bone marrow B cell apoptosis during in vivo influenza virus infection requires TNF-alpha and lymphotoxinalpha. J Immunol. 2002;169(11):6193-201.

30. Smith JN, Kanwar VS, MacNamara KC. Hematopoietic stem cell regulation by type I and II interferons in the pathogenesis of acquired aplastic anemia. Front Immunol. 2016;7:330.

31. Hirche C, et al. Systemic virus infections differentially modulate cell cycle state and functionality of long-term hematopoietic stem cells in vivo. Cell Rep. 2017;19(11):2345-56. This study highlights that acute as well as non-acute virus infections induce inflammatory responses within the bone marrow that affect LT-HSC phenotype and function. In these responses, IFN-I is an important, but not the only, crucial factor that signals hematopoietic stress and activates quiescent LT-HSCs.

32. Bordoni V, Bibas M, Viola D, Sacchi A, Cimini E, Tumino N, et al. Bone marrow CD34(+) progenitor cells from HIV-infected patients show an impaired $\mathrm{T}$ cell differentiation potential related to proinflammatory cytokines. AIDS Res Hum Retrovir. 2017;33(6): 590-6.

33. Bordoni V, Sacchi A, Cimini E, Casetti R, Tumino N, Ammassari A, et al. The different roles of interleukin 7 and interleukin 18 in affecting lymphoid hematopoietic progenitor cells and CD4 homeostasis in naive primary and chronic HIV-infected patients. Clin Infect Dis. 2016;63(12):1683-4.

34. Bordoni V, Viola D, Sacchi A, Pinnetti C, Casetti R, Cimini E, et al. IL-18 and stem cell factor affect hematopoietic progenitor cells in HIV-infected patients treated during primary HIV infection. Cytokine. 2018;103:34-7.

35. Huang C, Wang Y, Li X, Ren L, Zhao J, Hu Y, et al. Clinical features of patients infected with 2019 novel coronavirus in Wuhan, China. Lancet. 2020;395(10223):497-506.

36. Kadmon CS, Landers CT, Li HS, Watowich SS, Rodriguez A, King KY. MicroRNA-22 controls interferon alpha production and erythroid maturation in response to infectious stress in mice. Exp Hematol. 2017;56:7-15.

37. Hancock MH, Crawford LB, Pham AH, Mitchell J, Struthers HM, Yurochko AD, et al. Human cytomegalovirus miRNAs regulate TGF-beta to mediate myelosuppression while maintaining viral latency in $\mathrm{CD} 34(+)$ hematopoietic progenitor cells. Cell Host Microbe. 2020;27(1):104-14 e4.

38. Vainieri ML, et al. Systematic tracking of altered haematopoiesis during sporozoite-mediated malaria development reveals multiple response points. Open Biol. 2016;6(6). The authors describe a complex relationship between Plasmodium and host hematopoiesis and raise the question whether the variable responses observed may affect the outcome of the infection itself and its long-term consequences on the host.
39. Flach J, Bakker ST, Mohrin M, Conroy PC, Pietras EM, Reynaud $\mathrm{D}$, et al. Replication stress is a potent driver of functional decline in ageing haematopoietic stem cells. Nature. 2014;512(7513):198202.

40. Jaiswal S, Ebert BL. Clonal hematopoiesis in human aging and disease. Science. 2019;366(6465).

41. Montoya JG, Liesenfeld O. Toxoplasmosis. Lancet. 2004;363(9425):1965-76.

42. Czyzewski K, et al. Low seroprevalence and low incidence of infection with Toxoplasma gondii (Nicolle et Manceaux, 1908) in pediatric hematopoietic cell transplantation donors and recipients: Polish nationwide study. Folia Parasitol. 2019:66.

43. Petakov M, et al. Hematopoiesis during acute Toxoplasma gondii infection in mice. Haematologia (Budap). 2002;32(4):439-55.

44. Liu Q, Gao WW, Elsheikha HM, He JJ, Li FC, Yang WB, et al. Transcriptomic analysis reveals Toxoplasma gondii strain-specific differences in host cell response to dense granule protein GRA15. Parasitol Res. 2018;117(9):2785-93.

45. Wang P, Li S, Zhao Y, Zhang B, Li Y, Liu S, et al. The GRA15 protein from Toxoplasma gondii enhances host defense responses by activating the interferon stimulator STING. J Biol Chem. 2019;294(45):16494-508.

46. Roers A, Hiller B, Hornung V. Recognition of endogenous nucleic acids by the innate immune system. Immunity. 2016;44(4):739-54.

47. Kim H, Lee S, Lee SW. TRAF6 distinctly regulates hematopoietic stem and progenitors at different periods of development in mice. Mol Cell. 2018;41(8):753-61.

48. Ceesay MM, Desai SR, Berry L, Cleverley J, Kibbler CC, Pomplun S, et al. A comprehensive diagnostic approach using galactomannan, targeted beta-d-glucan, baseline computerized tomography and biopsy yields a significant burden of invasive fungal disease in at risk haematology patients. $\mathrm{Br} \mathrm{J}$ Haematol. 2015;168(2):219-29.

49. Neofytos D, Horn D, Anaissie E, Steinbach W, Olyaei A, Fishman $\mathrm{J}$, et al. Epidemiology and outcome of invasive fungal infection in adult hematopoietic stem cell transplant recipients: analysis of Multicenter Prospective Antifungal Therapy (PATH) Alliance registry. Clin Infect Dis. 2009;48(3):265-73.

50. Buckholz A, Kaplan A. Gastrointestinal mucormycosis presenting as emphysematous gastritis after stem cell transplant for myeloma. Mayo Clin Proc. 2020;95(1):33-4.

51. Hibbett DS, Binder M, Bischoff JF, Blackwell M, Cannon PF, Eriksson OE, et al. A higher-level phylogenetic classification of the fungi. Mycol Res. 2007;111(Pt 5):509-47.

52. Roden MM, Zaoutis TE, Buchanan WL, Knudsen TA, Sarkisova TA, Schaufele RL, et al. Epidemiology and outcome of zygomycosis: a review of 929 reported cases. Clin Infect Dis. 2005;41(5):634-53.

53. Espinosa $\mathrm{V}$, et al. Type III interferon is a critical regulator of innate antifungal immunity. Sci Immunol. 2017;2(16).

54. Griseri T, et al. Dysregulated hematopoietic stem and progenitor cell activity promotes interleukin-23-driven chronic intestinal inflammation. Immunity. 2012;37(6):1116-29. The authors identify HSPCs as a major target of the IL-23-driven inflammatory axis suggesting therapeutic strategies for the treatment of inflammatory bowel disease.

55. Griseri T, Arnold IC, Pearson C, Krausgruber T, Schiering C, Franchini F, et al. Granulocyte macrophage colony-stimulating factor-activated eosinophils promote interleukin-23 driven chronic colitis. Immunity. 2015;43(1):187-99.

56. Niu H, et al. The function of hematopoietic stem cells is altered by both genetic and inflammatory factors in lupus mice. Blood. 2013;121(11):1986-94. This elegant study shows that the loss of p18INK4c expression and an enhanced level of p21CIP1 in 
triple-congenic lupus-prone mice may protect HSCs from stress-induced depletion.

57. van der Valk FM, et al. Increased haematopoietic activity in patients with atherosclerosis. Eur Heart J. 2017;38(6):425-32.

58. Hernandez G, Mills TS, Rabe JL, Chavez JS, Kuldanek S, Kirkpatrick G, et al. Pro-inflammatory cytokine blockade attenuates myeloid expansion in a murine model of rheumatoid arthritis. Haematologica. 2020;105(3):585-97.

59. Barzaghi F, Amaya Hernandez LC, Neven B, Ricci S, Kucuk ZY, Bleesing JJ, et al. Long-term follow-up of IPEX syndrome patients after different therapeutic strategies: an international multicenter retrospective study. J Allergy Clin Immunol. 2018;141(3):103649 e5.

60. Kiykim A, Charbonnier LM, Akcay A, Karakoc-Aydiner E, Ozen A, Ozturk G, et al. Hematopoietic stem cell transplantation in patients with heterozygous STAT1 gain-of-function mutation. J Clin Immunol. 2019;39(1):37-44.

61. Alexander T, Farge D, Badoglio M, Lindsay JO, Muraro PA, Snowden JA, et al. Hematopoietic stem cell therapy for autoimmune diseases - clinical experience and mechanisms. J Autoimmun. 2018;92:35-46.

62. Kohler CA, et al. Peripheral cytokine and chemokine alterations in depression: a meta-analysis of 82 studies. Acta Psychiatr Scand. 2017;135(5):373-87.

63. Dowlati Y, Herrmann N, Swardfager W, Liu H, Sham L, Reim EK, et al. A meta-analysis of cytokines in major depression. Biol Psychiatry. 2010;67(5):446-57.

64. Khandaker GM, Pearson RM, Zammit S, Lewis G, Jones PB. Association of serum interleukin 6 and C-reactive protein in childhood with depression and psychosis in young adult life: a population-based longitudinal study. JAMA Psychiatry. 2014;71(10):1121-8.

65. Bachiller S, Jiménez-Ferrer I, Paulus A, Yang Y, Swanberg M, Deierborg T, et al. Microglia in neurological diseases: a road map to brain-disease dependent-inflammatory response. Front Cell Neurosci. 2018;12:488.

66. Fonseca-Pereira D, Arroz-Madeira S, Rodrigues-Campos M, Barbosa IAM, Domingues RG, Bento T, et al. The neurotrophic factor receptor RET drives haematopoietic stem cell survival and function. Nature. 2014;514(7520):98-101.

67. Yamazaki S, Ema H, Karlsson G, Yamaguchi T, Miyoshi H, Shioda $\mathrm{S}$, et al. Nonmyelinating Schwann cells maintain hematopoietic stem cell hibernation in the bone marrow niche. Cell. 2011;147(5):1146-58.

68. Lee J, et al. Causes and mechanisms of hematopoietic stem cell aging. Int J Mol Sci. 2019;20(6).

69. Beerman I, Bhattacharya D, Zandi S, Sigvardsson M, Weissman IL, Bryder D, et al. Functionally distinct hematopoietic stem cells modulate hematopoietic lineage potential during aging by a mechanism of clonal expansion. Proc Natl Acad Sci U S A. 2010;107(12):5465-70.

70. Rossi DJ, Bryder D, Seita J, Nussenzweig A, Hoeijmakers J, Weissman IL. Deficiencies in DNA damage repair limit the function of haematopoietic stem cells with age. Nature. 2007;447(7145):725-9.

71. Cohen HJ, et al. The association of plasma IL-6 levels with functional disability in community-dwelling elderly. J Gerontol A Biol Sci Med Sci. 1997;52(4):M201-8.

72. Maggio M, Basaria S, Ble A, Lauretani F, Bandinelli S, Ceda GP, et al. Correlation between testosterone and the inflammatory marker soluble interleukin-6 receptor in older men. J Clin Endocrinol Metab. 2006;91(1):345-7.

73. Bruunsgaard H, Andersen-Ranberg K, Jeune B, Pedersen AN, Skinhoj P, Pedersen BK. A high plasma concentration of TNFalpha is associated with dementia in centenarians. J Gerontol A Biol Sci Med Sci. 1999;54(7):M357-64.
74. Hasegawa Y, Sawada M, Ozaki N, Inagaki T, Suzumura A. Increased soluble tumor necrosis factor receptor levels in the serum of elderly people. Gerontology. 2000;46(4):185-8.

75. De Cecco M, et al. L1 drives IFN in senescent cells and promotes age-associated inflammation. Nature. 2019;566(7742):73-8.

76. King KY, et al. Environmental influences on clonal hematopoiesis. Exp Hematol. 2020;83:66-73. This is a comprehensive review that describes the different environmental factors that promote the appearance of clonal hematopoiesis.

77. Zhang H, Nguyen-Jackson H, Panopoulos AD, Li HS, Murray PJ, Watowich SS. STAT3 controls myeloid progenitor growth during emergency granulopoiesis. Blood. 2010;116(14):2462-71.

78. McKelvey KJ, Hudson AL, Back M, Eade T, Diakos CI. Radiation, inflammation and the immune response in cancer. Mamm Genome. 2018;29(11-12):843-65.

79. Kadhim MA, Lorimore SA, Townsend KMS, Goodhead DT, Buckle VJ, Wright EG. Radiation-induced genomic instability: delayed cytogenetic aberrations and apoptosis in primary human bone marrow cells. Int J Radiat Biol. 1995;67(3):287-93.

80. Rodman C, et al. In vitro and in vivo assessment of direct effects of simulated solar and galactic cosmic radiation on human hematopoietic stem/progenitor cells. Leukemia. 2017;31(6):1398-407. The authors demonstrate that galactic cosmic ray leads to mutation in genes involved in hematopoiesis and abnormal development of T-ALL.

81. Almeida-Porada G, Rodman C, Kuhlman B, Brudvik E, Moon J, George S, et al. Exposure of the bone marrow microenvironment to simulated solar and galactic cosmic radiation induces biological bystander effects on human hematopoiesis. Stem Cells Dev. 2018;27(18):1237-56.

82. van Lunzen J, Altfeld M. Sex differences in infectious diseasescommon but neglected. J Infect Dis. 2014;209(Suppl 3):S79-80.

83. Powell B, Szleifer I, Dhaher YY. In silico study of principal sex hormone effects on post-injury synovial inflammatory response. PLoS One. 2018;13(12):e0209582.

84.• Nakada D, et al. Oestrogen increases haematopoietic stem-cell self-renewal in females and during pregnancy. Nature. 2014;505(7484):555-8. This study shows that estrogen induces hematopoietic stem cell (HSC) self-renewal through its interaction with estrogen receptor alpha.

85. Pietras EM. Inflammation: a key regulator of hematopoietic stem cell fate in health and disease. Blood. 2017;130(15):1693-8.

86. Yu WM, Liu X, Shen J, Jovanovic O, Pohl EE, Gerson SL, et al. Metabolic regulation by the mitochondrial phosphatase PTPMT1 is required for hematopoietic stem cell differentiation. Cell Stem Cell. 2013;12(1):62-74.

87. Takubo K, Nagamatsu G, Kobayashi CI, Nakamura-Ishizu A, Kobayashi H, Ikeda E, et al. Regulation of glycolysis by Pdk functions as a metabolic checkpoint for cell cycle quiescence in hematopoietic stem cells. Cell Stem Cell. 2013;12(1):49-61.

88. Ito K, Carracedo A, Weiss D, Arai F, Ala U, Avigan DE, et al. A PML-PPAR-delta pathway for fatty acid oxidation regulates hematopoietic stem cell maintenance. Nat Med. 2012;18(9):1350-8.

89. Guillemot-Legris O, Masquelier J, Everard A, Cani PD, Alhouayek M, Muccioli GG. High-fat diet feeding differentially affects the development of inflammation in the central nervous system. J Neuroinflammation. 2016;13(1):206.

90. Duan Y, Zeng L, Zheng C, Song B, Li F, Kong X, et al. Inflammatory links between high fat diets and diseases. Front Immunol. 2018;9:2649.

91. Tall AR, Yvan-Charvet L. Cholesterol, inflammation and innate immunity. Nat Rev Immunol. 2015;15(2):104-16.

92. Yvan-Charvet L, Pagler T, Gautier EL, Avagyan S, Siry RL, Han $\mathrm{S}$, et al. ATP-binding cassette transporters and HDL suppress hematopoietic stem cell proliferation. Science. 2010;328(5986): 1689-93. 
93. Tang Y, Liu W, Wang W, Fidler T, Woods B, Levine RL, et al. Inhibition of JAK2 suppresses myelopoiesis and atherosclerosis in Apoe(-/-) mice. Cardiovasc Drugs Ther. 2020;34:145-52.

94. Taya Y, Ota Y, Wilkinson AC, Kanazawa A, Watarai H, Kasai M, et al. Depleting dietary valine permits nonmyeloablative mouse hematopoietic stem cell transplantation. Science. 2016;354(6316):1152-5.

95. Ueland PM, McCann A, Midttun Ø, Ulvik A. Inflammation, vitamin B6 and related pathways. Mol Asp Med. 2017;53:10-27.

96. Ellulu MS, et al. Effect of vitamin $\mathrm{C}$ on inflammation and metabolic markers in hypertensive and/or diabetic obese adults: a randomized controlled trial. Drug Des Dev Ther. 2015;9:3405-12.

97. Cortes M, Chen MJ, Stachura DL, Liu SY, Kwan W, Wright F, et al. Developmental vitamin D availability impacts hematopoietic stem cell production. Cell Rep. 2016;17(2):458-68.

98. Farhadfar N, Kelly DL, Mead L, Nair S, Colee J, Irizarry Gatell V, et al. Dietary intake and diet quality of hematopoietic stem cell transplantation survivors. Biol Blood Marrow Transplant. 2020;26:1154-9.

99. Xia S, Li XP, Cheng L, Han MT, Zhang MM, Shao QX, et al. Fish oil-rich diet promotes hematopoiesis and alters hematopoietic niche. Endocrinology. 2015;156(8):2821-30.

100. Cheng CW, et al. Prolonged fasting reduces IGF-1/PKA to promote hematopoietic-stem-cell-based regeneration and reverse immunosuppression. Cell Stem Cell. 2014;14(6):810-23. This work provides evidence that 48 -h cycles of fasting stimulate HSC regeneration in mice by inhibiting insulin growing factor 1 (IGF1) signaling.

101. Ferraro F, et al. Diabetes impairs hematopoietic stem cell mobilization by altering niche function. Sci Transl Med. 2011;3(104): 104ra101.

102.• Frodermann V, et al. Exercise reduces inflammatory cell production and cardiovascular inflammation via instruction of hematopoietic progenitor cells. Nat Med. 2019;25(11):1761-71. This elegant work provides evidence that physical activity protects mice from leukocytosis by decreasing hematopoietic activity.

103. Emmons R, Xu G, Hernández-Saavedra D, Kriska A, Pan YX, Chen $\mathrm{H}$, et al. Effects of obesity and exercise on colon cancer induction and hematopoiesis in mice. Am J Physiol Endocrinol Metab. 2019;316(2):E210-20.

104. Khaldoyanidi S, Sikora L, Orlovskaya I, Matrosova V, Kozlov V, Sriramarao P. Correlation between nicotine-induced inhibition of hematopoiesis and decreased CD44 expression on bone marrow stromal cells. Blood. 2001;98(2):303-12.

105. Siggins RW, Hossain F, Rehman T, Melvan JN, Zhang P, Welsh DA. Cigarette smoke alters the hematopoietic stem cell niche. Med Sci (Basel). 2014;2(1):37-50.

106. Pandit TS, Sikora L, Muralidhar G, Rao SP, Sriramarao P. Sustained exposure to nicotine leads to extramedullary hematopoiesis in the spleen. Stem Cells. 2006;24(11):2373-81.

107.• Cyprus GN, et al. Cigarette smoke increases pro-inflammatory markers and inhibits osteogenic differentiation in experimental exposure model. Acta Biomater. 2018;76:308-18. The authors demonstrate that cigarette smoke exposure leads to decreased bone marrow formation and the production of antiinflammatory interleukin but increased pro-inflammatory interleukin.

108. Shi H, Shao X, Hong Y. Association between cigarette smoking and the susceptibility of acute myeloid leukemia: a systematic review and meta-analysis. Eur Rev Med Pharmacol Sci. 2019;23(22):10049-57. This is a comprehensive review of the role of microbiota in hematopoiesis in the bone marrow.

109. Yan H, Baldridge MT, King KY. Hematopoiesis and the bacterial microbiome. Blood. 2018;132(6):559-64.

110. Balmer ML, Schürch CM, Saito Y, Geuking MB, Li H, Cuenca $\mathrm{M}$, et al. Microbiota-derived compounds drive steady-state granulopoiesis via MyD88/TICAM signaling. J Immunol. 2014;193(10):5273-83.

111. Khosravi A, Yáñez A, Price JG, Chow A, Merad M, Goodridge HS, et al. Gut microbiota promote hematopoiesis to control bacterial infection. Cell Host Microbe. 2014;15(3):374-81.

112.• Josefsdottir KS, et al. Antibiotics impair murine hematopoiesis by depleting the intestinal microbiota. Blood. 2017;129(6):729-39. First demonstration that antibiotic-treated mice show the depletion of microbiota that promotes decreased HSCs and multipotent progenitors through STAT1 signaling.

113. Iwamura $\mathrm{C}$, et al. Sensing of the microbiota by NOD1 in mesenchymal stromal cells regulates murine hematopoiesis. Blood. 2017;129(2):171-6. The authors propose that NOD1 signaling in MSCs serves as an important pathway underlying the requirement for microbiota in the maintenance of steady-state hematopoiesis.

114. Inagaki H, Suzuki T, Nomoto K, Yoshikai Y. Increased susceptibility to primary infection with Listeria monocytogenes in germfree mice may be due to lack of accumulation of L-selectin+ CD44+ $\mathrm{T}$ cells in sites of inflammation. Infect Immun. 1996;64(8):3280-7.

115. Tada T, Yamamura S, Kuwano Y, Abo T. Level of myelopoiesis in the bone marrow is influenced by intestinal flora. Cell Immunol. 1996;173(1):155-61.

116. Staffas A, Burgos da Silva M, Slingerland AE, Lazrak A, Bare CJ, Holman CD, et al. Nutritional support from the intestinal microbiota improves hematopoietic reconstitution after bone marrow transplantation in mice. Cell Host Microbe. 2018;23(4):447-57 e4.

117. Lee S, Kim H, You G, Kim YM, Lee S, le VH, et al. Bone marrow CX3CR1+ mononuclear cells relay a systemic microbiota signal to control hematopoietic progenitors in mice. Blood. 2019;134(16):1312-22.

118. Hirai H, Zhang P, Dayaram T, Hetherington CJ, Mizuno SI, Imanishi $\mathrm{J}$, et al. C/EBPbeta is required for 'emergency' granulopoiesis. Nat Immunol. 2006;7(7):732-9.

119. Wilson A, Laurenti E, Oser G, van der Wath RC, Blanco-Bose W, Jaworski M, et al. Hematopoietic stem cells reversibly switch from dormancy to self-renewal during homeostasis and repair. Cell. 2008;135(6):1118-29.

120. Greenbaum AM, Link DC. Mechanisms of G-CSF-mediated hematopoietic stem and progenitor mobilization. Leukemia. 2011;25(2):211-7. In this review, the authors discuss the mechanisms by which granulocyte colony-stimulating factor (GCSF) induces HSPC mobilization.

121. Manz MG, Boettcher S. Emergency granulopoiesis. Nat Rev Immunol. 2014;14(5):302-14.

122. Croker BA, Metcalf D, Robb L, Wei W, Mifsud S, DiRago L, et al. SOCS3 is a critical physiological negative regulator of GCSF signaling and emergency granulopoiesis. Immunity. 2004;20(2):153-65.

123. Regan-Komito D, Swann JW, Demetriou P, Cohen ES, Horwood NJ, Sansom SN, et al. GM-CSF drives dysregulated hematopoietic stem cell activity and pathogenic extramedullary myelopoiesis in experimental spondyloarthritis. Nat Commun. 2020;11(1):155.

124. Mitroulis I, Ruppova K, Wang B, Chen LS, Grzybek M, Grinenko $\mathrm{T}$, et al. Modulation of myelopoiesis progenitors is an integral component of trained immunity. Cell. 2018;172(1-2):147-61 e12.

125. Mossadegh-Keller N, Sarrazin S, Kandalla PK, Espinosa L, Stanley ER, Nutt SL, et al. M-CSF instructs myeloid lineage fate in single haematopoietic stem cells. Nature. 2013;497(7448):239 43.

126. Auffray C, Sieweke MH, Geissmann F. Blood monocytes: development, heterogeneity, and relationship with dendritic cells. Annu Rev Immunol. 2009;27:669-92. 
127. de Bruin AM, Demirel Ö, Hooibrink B, Brandts CH, Nolte MA. Interferon-gamma impairs proliferation of hematopoietic stem cells in mice. Blood. 2013;121(18):3578-85.

128. Zhang CRC, Nix D, Gregory M, Ciorba MA, Ostrander EL, Newberry RD, et al. Inflammatory cytokines promote clonal hematopoiesis with specific mutations in ulcerative colitis patients. Exp Hematol. 2019;80:36-41 e3.

129. Lin FC, Karwan M, Saleh B, Hodge DL, Chan T, Boelte KC, et al. IFN-gamma causes aplastic anemia by altering hematopoietic stem/progenitor cell composition and disrupting lineage differentiation. Blood. 2014;124(25):3699-708.

130. Bihari C, Anand L, Rooge S, Kumar D, Saxena P, Shubham S, et al. Bone marrow stem cells and their niche components are adversely affected in advanced cirrhosis of the liver. Hepatology. 2016;64(4):1273-88.

131. Zoller EE, Lykens JE, Terrell CE, Aliberti J, Filipovich AH, Henson PM, et al. Hemophagocytosis causes a consumptive anemia of inflammation. J Exp Med. 2011;208(6):1203-14.

132. Jordan MB, Hildeman D, Kappler J, Marrack P. An animal model of hemophagocytic lymphohistiocytosis (HLH): CD8+ T cells and interferon gamma are essential for the disorder. Blood. 2004;104(3):735-43.

133. Mojic M, Takeda K, Hayakawa Y. The dark side of IFN-gamma: its role in promoting cancer immunoevasion. Int J Mol Sci. 2017;19(1)

134. Kaufmann E, Sanz J, Dunn JL, Khan N, Mendonça LE, Pacis A, et al. BCG educates hematopoietic stem cells to generate protective innate immunity against tuberculosis. Cell. 2018;172(1-2): 176-90 e19.

135. Paludan SR, Reinert LS, Hornung V. DNA-stimulated cell death: implications for host defence, inflammatory diseases and cancer. Nat Rev Immunol. 2019;19(3):141-53.

136.• Essers MA, et al. IFNalpha activates dormant haematopoietic stem cells in vivo. Nature. 2009;458(7240):904-8. In this relevant study, the authors demonstrate that acute IFNa injections promote HSC proliferation while chronic treatment functionally compromised HSCs.

137. Walter D, et al. Exit from dormancy provokes DNA-damageinduced attrition in haematopoietic stem cells. Nature. 2015;520(7548):549-52. This study establishes a novel link between physiological stress and DNA damage in normal HSCs and provides a mechanistic explanation for the universal accumulation of DNA damage in HSCs during aging and the accelerated failure of the hematopoietic system in Fanconi anemia patients.

138. Haas S, et al. Inflammation-induced emergency megakaryopoiesis driven by hematopoietic stem cell-like megakaryocyte progenitors. Cell Stem Cell. 2015;17(4):422-34. This elegant study reveals an emergency machinery that counteracts lifethreatening platelet depletions during acute inflammation.

139. Sato T, et al. Interferon regulatory factor-2 protects quiescent hematopoietic stem cells from type I interferon-dependent exhaustion. Nat Med. 2009;15(6):696-700 These findings provide insight into the molecular basis for the maintenance of HSC quiescence.

140. Huerga Encabo $\mathrm{H}$, et al. The transcription factor NFAT5 limits infection-induced type I interferon responses. J Exp Med. 2020;217(3)

141. Pietras EM, et al. Re-entry into quiescence protects hematopoietic stem cells from the killing effect of chronic exposure to type I interferons. J Exp Med. 2014;211(2):245-62. The authors reveal that IFN-1s can poise HSCs for apoptosis but induce direct cell killing only upon active proliferation.

142. de Laval B, et al. C/EBPbeta-dependent epigenetic memory induces trained immunity in hematopoietic stem cells. Cell Stem Cell. 2020.

143. Franchi L, Warner N, Viani K, Nuñez G. Function of Nod-like receptors in microbial recognition and host defense. Immunol Rev. 2009;227(1):106-28.

144. Pronk CJ, et al. Tumor necrosis factor restricts hematopoietic stem cell activity in mice: involvement of two distinct receptors. J Exp Med. 2011;208(8):1563-70.

145. Etzrodt M, Ahmed N, Hoppe PS, Loeffler D, Skylaki S, Hilsenbeck $\mathrm{O}$, et al. Inflammatory signals directly instruct PU.1 in HSCs via TNF. Blood. 2019;133(8):816-9.

146. Van Gorp H, Lamkanfi M. The emerging roles of inflammasomedependent cytokines in cancer development. EMBO Rep. 2019;20(6).

147. Venkatesha SH, Dudics S, Weingartner E, So EC, Pedra JHF, Moudgil KD. Altered Th17/Treg balance and dysregulated IL1 beta response influence susceptibility/resistance to experimental autoimmune arthritis. Int J Immunopathol Pharmacol. 2015;28(3): 318-28.

148. Pietras EM, Mirantes-Barbeito C, Fong S, Loeffler D, Kovtonyuk LV, Zhang SY, et al. Chronic interleukin-1 exposure drives haematopoietic stem cells towards precocious myeloid differentiation at the expense of self-renewal. Nat Cell Biol. 2016;18(6): 607-18.

149. Meisel M, Hinterleitner R, Pacis A, Chen L, Earley ZM, Mayassi $\mathrm{T}$, et al. Microbial signals drive pre-leukaemic myeloproliferation in a Tet2-deficient host. Nature. 2018;557(7706):580-4.

150. Zhao JL, Ma C, O'Connell RM, Mehta A, DiLoreto R, Heath JR, et al. Conversion of danger signals into cytokine signals by hematopoietic stem and progenitor cells for regulation of stress-induced hematopoiesis. Cell Stem Cell. 2014;14(4):445-59.

151. Cai, Z., Kotzin J.J., Ramdas B., Chen S., Nelanuthala S., Palam L.R., Pandey R., Mali R.S., Liu Y., Kelley M.R., Sandusky G., Mohseni M., Williams A., Henao-Mejia J., Kapur R., Inhibition of inflammatory signaling in Tet2 mutant preleukemic cells mitigates stress-induced abnormalities and clonal hematopoiesis. Cell Stem Cell, 2018. 23(6): p. 833-849 e5.

Publisher's Note Springer Nature remains neutral with regard to jurisdictional claims in published maps and institutional affiliations. 